\title{
Pseudotumor Orbitário em Paciente com Doença de Still do Adulto: Associação Incomum
}

\section{Orbital Pseudo Tumor in a Patient with Adult-onset Still's Disease: Uncommom Association}

\author{
Márcia Regina Rosa Scalcon ${ }^{(1)}$, Viviane Roseli da Cunha ${ }^{(2)}$, Álvaro Garcia Rossi ${ }^{(3)}$, Eduardo dos Santos Paiva ${ }^{(4)}$
}

\section{RESUMO}

A doença de Still do adulto é uma desordem inflamatória rara, caracterizada por picos febris diários, rash evanescente, artrite e envolvimento de múltiplos órgãos. Manifestações oculares têm sido descritas associadas às manifestações clínicas clássicas da doença. Será descrito um caso de pseudotumor orbitário, condição clínica incomum, em uma paciente com diagnóstico de doença de Still do adulto.

Palavras-chave: doença de Still do adulto, pseudotumor orbitário, artrite, proptose.

\section{INTRODUÇÃO}

A doença de Still do adulto (DSA) é uma desordem inflamatória sistêmica rara, de etiologia desconhecida, caracterizada por picos febris diários, erupção cutânea evanescente de coloração salmão, artrite e envolvimento de múltiplos órgãos ${ }^{(1)}$.

Manifestações oculares estão descritas na DSA, entre elas: síndrome sicca, exsudatos retinianos, irite, panoftalmite, síndrome de Brown e pseudotumor orbitário inflamatório ${ }^{(2,3)}$.

O pseudotumor orbitário inflamatório é uma doença inflamatória idiopática benigna que pode afetar qualquer estrutura da órbita, sendo comuns sintomas como dor, proptose, sinais e sintomas inflamatórios locais. Sua etiologia é desconhecida, porém há provável processo imunomediado associado à sua patogênese. Reconhecimento precoce dessa condição clínica é fundamental, uma vez que pode apresentar curso clinicamente maligno, com perda da visão e disfunção oculomotora.

\begin{abstract}
Adult-onset Still's Disease is a rare systemic inflammatory disorder characterized by quotidian spiking fevers, evanescent rash, arthritis, and multiorgan involvement. Ocular manifestations have been reported associated with the classical clinical findings of this disease. We describe a case of orbital pseudo tumor, an uncommon clinical condition, in a patient with diagnosis of adult-onset Still's Disease.
\end{abstract}

Keywords: adult-onset Still's Disease, orbital pseudo tumor, arthritis, proptosis.

\section{RELATO DE CASO}

Paciente do sexo feminino, 55 anos, parda, sem comorbidades exceto hipertensão arterial, foi admitida por rash pruriginoso, artrite e picos febris.

A paciente estava assintomática até dois meses antes da internação, quando iniciou com exantema urticariforme em região anterior cervical e torácica alta, evanescente, acompanhado de calafrios e febre de até $39^{\circ} \mathrm{C}$, predominantemente vespertina e noturna. Um mês após o quadro inicial, passou a apresentar artrite simétrica em joelhos, punhos, metacarpofalangeanas e ombros com resposta parcial ao uso de antiinflamatórios não-esteroidais (AINES).

Ao exame físico apresentava-se ligeiramente descorada, com ausculta cardíaca e respiratória sem alterações e abdome globoso, sem organomegalias. Não havia linfonodos palpáveis e observava-se artrite em joelhos, punhos e segunda e terceira metacarpofalangeanas à esquerda.

A investigação laboratorial mostrou leucócitos de $26.000 / \mathrm{mm}^{3}$ (polimorfonucleares $86 \%$ e linfócitos $7 \%$ );

Recebido em 8/4/2008. Aprovado, após revisão, em 21/8/2008. Declaramos a inexistência de conflitos de interesse

1. Especializanda do curso de especialização em Reumatologia do Hospital de Clínicas da Universidade Federal do Paraná (UFPR)

2. Médica Reumatologista da Universidade Federal de Santa Maria (UFSM).

3. Professor assistente da disciplina de Oftalmologia da UFSM.

4. Professor assistente da disciplina de Reumatologia da UFPR

Endereço para correspondência: Márcia Regina Rosa Scalcon, Rua Presidente Carlos Cavalcanti, 649/51, 80510-040, Curitiba, PR, e-mail: marciascalcon@terra.com.br. 
hemoglobina 10,6 g/dL; plaquetas $521.000 / \mathrm{mm}^{3}$; velocidade de hemossedimentação (VHS) $100 \mathrm{~mm}$; DHL $667 \mathrm{U} / \mathrm{L}$ (normal 135-214); fosfatase alcalina $422 \mathrm{U} / \mathrm{L}$ (normal 32-104); TGO $52 \mathrm{U} / \mathrm{L}$ (normal até 31); TGP 86 $\mathrm{U} / \mathrm{L}$ (normal até 33); uréia, creatinina, função tireoidiana e exame qualitativo de urina normais e ferritina de $5250 \mu \mathrm{g} / \mathrm{L}$ (normal 10-200). Fator antinuclear, fator reumatóide e testes para síndrome da imunodeficiência humana e hepatites foram negativos. O ultra-som de abdome, a radiografia de tórax, o ecocardiograma transesofágico e o exame ginecológico foram normais. A urocultura e as hemoculturas foram negativas.

Excluídas outras hipóteses para o quadro clínico, fez-se então o diagnóstico provisório de doença de Still do adulto, sendo então iniciada terapia com prednisona $20 \mathrm{mg} /$ dia. No seguimento ambulatorial, houve necessidade do uso de hidroxicloroquina e metotrexato para o controle da artrite.

Oito meses após o diagnóstico inicial, a paciente apresentou edema endurecido na região lateral da pálpebra superior direita, doloroso, associado à visão turva esporádica e lacrimejamento (Figuras l e 2). A tomografia computadorizada (TC) e a ressonância nuclear magnética (RNM) de órbitas evidenciaram espessamento de partes moles periorbitária à direita, aumento do volume e indefinição da glândula lacrimal ipsilateral e borramento da gordura adjacente, sugestivos de pseudotumor inflamatório (Figura 3).

Foi submetida à biópsia da lesão periorbitária, cujo exame anatomopatológico evidenciou amostras de tecido fibroadiposo e muscular com infiltrado linfocitário perivascular, por vezes configurando agregados linfóides. Não houve representação de glândula lacrimal na amostra.

Definida a origem inflamatória do material da biópsia e devido ao quadro ocular exuberante, optou-se por pulsoterapia de metilprednisolona durante três dias, seguida de prednisona $60 \mathrm{mg} /$ dia, com redução gradual da dosagem.

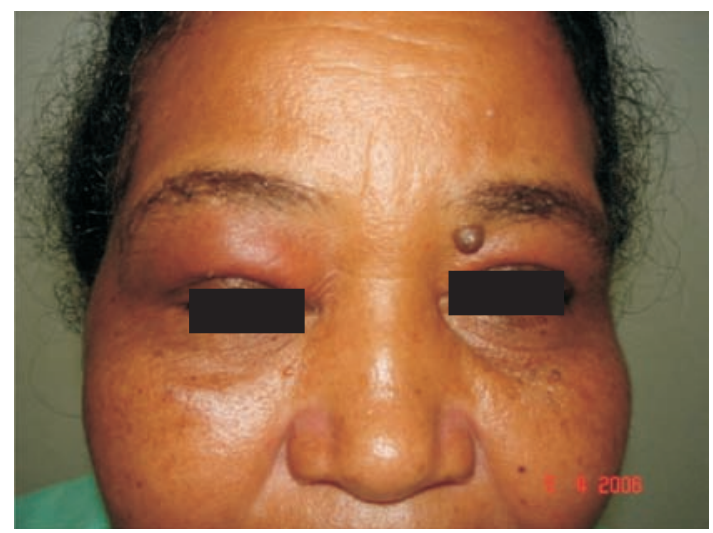

Figura 1 - Paciente com doença de Still do adulto e pseudotumor orbitário inflamatório à direita.

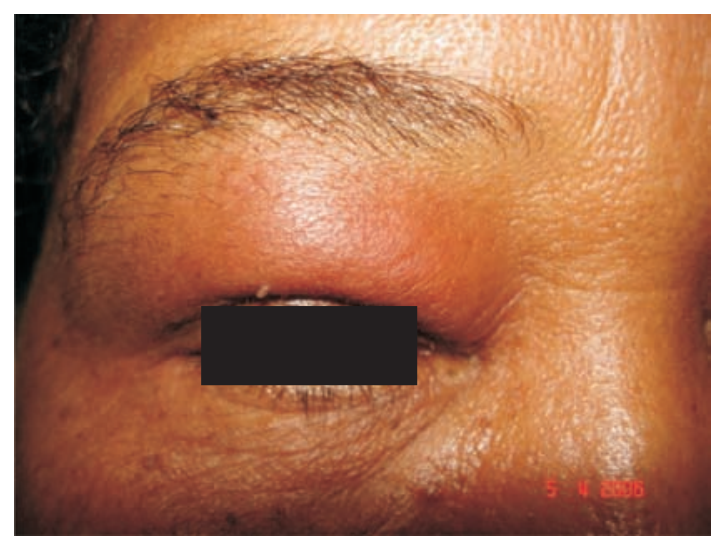

Figura 2 - Aumento de volume e eritema em pálpebra superior direita correspondente a pseudotumor orbitário inflamatório.

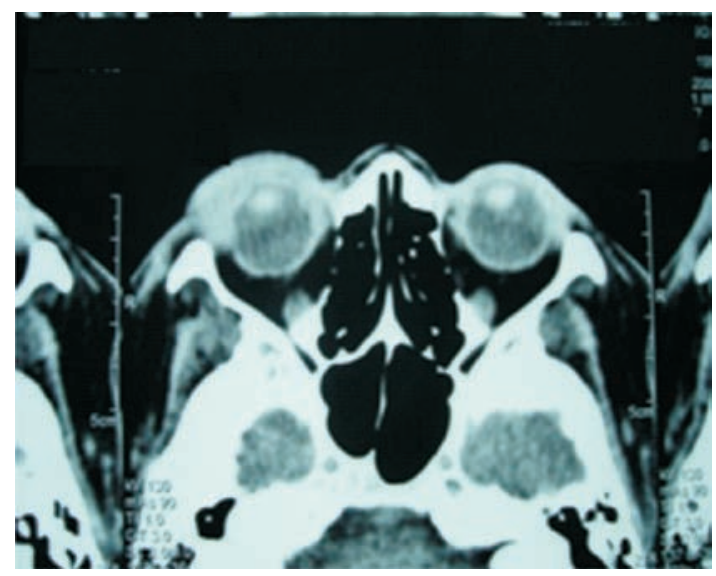

Figura 3 - TC de órbitas mostrando espessamento de partes moles periorbitária à direita, aumento e indefinição da glândula lacrimal e borramento da gordura adjacente.

No décimo dia pós-pulso, a paciente apresentava melhora importante do edema palpebral, com recuperação quase completa dos movimentos oculares (Figura 4).

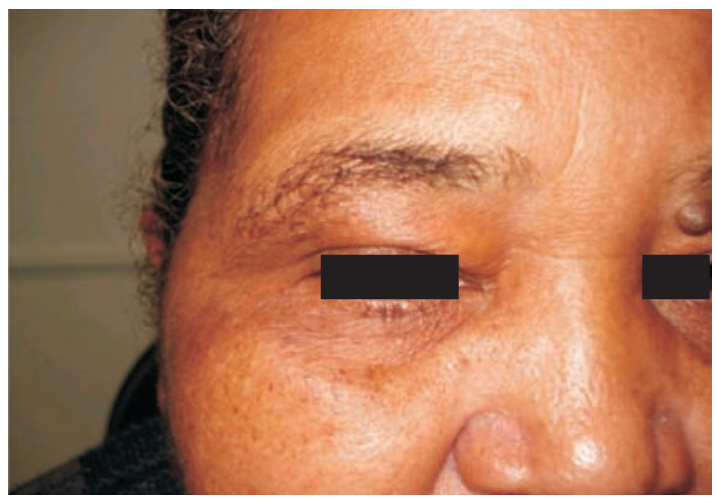

Figura 4 - Paciente 30 dias após corticoterapia com resolução completa do quadro inflamatório ocular. 


\section{DISCUSSÃO}

A paciente do presente relato apresentou doença multissistêmica, caracterizada por febre alta diária, rash cutâneo evanescente, com provas inflamatórias elevadas, poliartrite, leucocitose, trombocitose, aumento de enzimas hepáticas e da ferritina. Afastadas as possibilidades diagnósticas de infecção, neoplasia ou outras doenças reumatológicas, fez-se o diagnóstico de doença de Still do adulto.

A doença de Still do adulto permanece um diagnóstico de exclusão ${ }^{(3,4)}$, com diagnóstico diferencial amplo, incluindo infecções virais (rubéola, citomegalovírus, Epstein-Barr, caxumba, Coksakie e adenovírus) ${ }^{(4)}$, além de desordens neoplásicas (leucemia, linfoma e linfadenopatia angioblástica) ${ }^{(4)}$. Pode também ser confundida com artrite reativa (além de outras espondiloartropatias), síndrome hemofagocítica, dermatomiosite, síndrome de Kikuchi, síndrome de Sweet, desordens granulomatosas e vasculites ${ }^{(4)}$.

As manifestações clínicas mais comuns da DSA incluem artrite e artralgia, mialgia, febre e calafrios, erupção cutânea, aumento de linfonodos, hepatoesplenomegalia, dor abdominal, serosite e faringite não-exsudativa ${ }^{(2,5)}$.

Durante o seguimento clínico, a paciente desenvolveu manifestação incomum da doença de Still do adulto - o pseudotumor orbitário inflamatório.

O pseudotumor orbitário inflamatório ou inflamação orbital idiopática foi descrito pela primeira vez por Gleason, em $1903^{(6)} \mathrm{e}$, posteriormente, nomeado por Birch-Hirschfeld em 1930 ${ }^{(7)}$, como doença inflamatória idiopática benigna que pode afetar qualquer estrutura da órbita ${ }^{(8)}$. O acometimento ocular varia desde processo difuso a comprometimento focal de tecidos específicos da órbita, como glândula lacrimal, músculos extraoculares e gordura orbital ${ }^{(9,10)}$.

Clinicamente, é comum ocorrer dor, proptose, sinais e sintomas inflamatórios, como eritema e edema local. De acordo com a localização acometida, pode suceder fibrose, efeito de massa, ptose, quemose, disfunção da motilidade dos músculos extraoculares (associado à diplopia) e neuropatia óptica ${ }^{(9,10)}$. A apresentação é tipicamente unilateral, mas o acometimento bilateral não é incomum ${ }^{(9)}$.

A patogênese ainda não é definida, porém várias linhas de evidência apontam para provável processo imunomediado. Reforçando este fato, há relatos de associação de pseudotumor orbitário com desordens imunológicas sistêmicas, como doença de Crohnn ${ }^{(11)}$, lúpus eritematoso sistêmico (LES) $)^{(12)}$, artrite reumatóide (AR) ${ }^{(13)}$, miastenia gravis $^{14)}$, entre outras. O pseudotumor orbitário tipicamen- te responde, de modo favorável, ao uso de corticoesteróides sistêmicos e o sucesso terapêutico tem sido relatado com agentes imunossupressores, como ciclosporina, metotrexato e ciclofosfamida ${ }^{(9)}$.

$\mathrm{Na}$ TC, o pseudotumor orbitário inflamatório é visto como massa difusa ou focal pobremente realçada pelo contraste. No caso do envolvimento da glândula lacrimal, o aumento difuso da glândula com preservação da sua forma pode ser acompanhada por reação inflamatória do tecido periglandular, com borramento da sua margem ${ }^{(9,10)}$.

O espectro histopatológico é tipicamente amplo, variando de infiltrado polimórfico difuso típico à inflamação granulomatosa atípica, eosinofilia tissular e esclerose infiltrativa ${ }^{(9)}$. No entanto, a histopatologia clássica envolve infiltrado de linfócitos esparsos, células plasmáticas e histiócitos, com preservação da arquitetura do tecido afetado ${ }^{(15)}$. Alguns autores consideram a forma esclerosante como estágio terminal de um continumm histopatológico do pseudotumor orbitário, caracterizado por componente maior de linfócitos no estágio inicial, passando para uma fase de fibrose progressiva, em um estágio mais crônico ${ }^{(16)}$. Outros, entretanto, acreditam se tratar de doenças distintas ${ }^{(17)}$.

A inflamação orbital idiopática pode ocorrer isoladamente ou em associação com várias doenças agudas e crônicas. Condições clínicas associadas incluem infecção viral de via aérea superior, faringite estreptocócica, LES, amiloidose, artrite psoriática, doença de Lyme, miocardite de células gigantes, doença de Crohn, doença de Behçet, doença de Still do adulto, artrite reumatóide (AR), asma alérgica, entre outras ${ }^{(9,10,15)}$

Fazem parte do diagnóstico diferencial doenças oculares da tireóide, celulite orbital, congestão vascular, infiltração metastática, linfoma, doença granulomatosa e vasculite ${ }^{(8)}$.

As opções terapêuticas são variadas e incluem a observação clínica, antiinflamatórios não-esteroidais (AINES), radiação, cirurgia, imunoglobulina IV, plasmaferese, corticoesteróides, imunossupressores e agentes biológicos (infliximabe) $)^{(10,15)}$. Em casos leves, a observação simples ou o uso de AINES é suficiente ${ }^{(15)}$. No entanto, corticoesteróides permanecem a primeira linha de tratamento da maioria dos casos de pseudotumor orbitário ${ }^{(9)}$, ficando as demais opções reservadas para casos refratários, recidivas ou intolerância ao uso dos corticoesteróides.

A despeito do resultado geralmente favorável com o uso de corticoesteróides, recidivas e inflamação persistente podem complicar o curso clínico e o tratamento ${ }^{(9)}$.

A relação entre doença de Still do adulto e pseudotumor orbitário é pouco relatada na literatura. Cush, Leibowitz e 
Friedman ${ }^{(18)}$ descreveram um paciente do sexo masculino, de 21 anos, com doença de Still do adulto que apresentou dificuldade de abertura ocular, diplopia, ptose, dor e edema periorbital, caracterizado como pseudotumor orbitário, com boa resposta terapêutica com corticoesteróide. Outro relato foi feito por Ganday et al. ${ }^{(19)}$ que descreveram uma paciente de 29 anos com doença de Still do adulto que desenvolveu diplopia transitória e paralisia do terceiro nervo craniano.

\section{REFERÊNCIAS}

1. Efthimiou P, Georgy S: Pathogenesis and management of adultonset Still's disease. Semin Arthritis Rheum 36: 144-52, 2006.

2. Estaile JM: Adult still'disease. Rheumatology $3^{\mathrm{a}}$ ed. p. 795, 2003.

3. Appenzeller S, Castro GW, Costallat LTL, Samara AM, Bértolo MB: Doença de Still do adulto: diagnóstico e evolução. Rev Bras Reumatol 43(6): 352-7, 2003.

4. Efthimiou P, Paik PK, Bielory L: Diagnosis and management of adult onset Still's disease. Ann Rheum Dis 65: 564-72, 2006.

5. Kádár J, Petrovicz E: Adult-onset Still's disease. Best Pract Res Clin Rheumatol 18(5): 663-76, 2004.

6. Gleason JE: Idiopathic myositis involving the intraocular muscles. Ophthalmol Rec 12: 471-8, 1903.

7. Birch-Hirschfeld A: Zur diagnostic and pathologic der orbital tumoren. Ber Zusammenkunft Dtsch Ophthalmol Ges 32: 127 35,1905

8. Stidham DB, Sondhi N, Plager D, Helveston E: Presumed Isolated Inflammation of the superior oblique muscle in idiopatic orbital myositis. Ophthalmology 105(12): 2216-9, 1998.

9. Yuen SJA, Rubin P: Idiopathic orbital inflammation: distribution, clinical features, and treatment outcome. Arch Ophthalmol 121: 491-9, 2003.

10. Lutt JR, Lim LL, Phal PM, Rosenbaum JT: Orbital inflammatory disease. Semin Arthritis Rheum 37: 207-22, 2008
Portanto, não obstante a associação rara, o diagnóstico de pseudotumor orbitário deve ser considerado em pacientes com doença de Still do adulto confirmada, que apresentem alteração oftalmológica recente. Como o pseudotumor pode assumir prognóstico ocular reservado, o diagnóstico correto se faz necessário para orientar a terapêutica e evitar danos, como perda da visão e disfunção oculomotora $^{(9)}$.

11. Squires Jr RH, Zwiener RJ, Kennedy RH: Orbital myositis and Crohn's disease. J Pediatr Gastroenterol Nutr 15: 448-51, 1992.

12. Serop S, Vianna RN, Claeys M, De Laey JJ: Orbital myositis secondary to systemic lupus erythematosus. Acta Ophthalmol (Copenh) 72: 520-3, 1994.

13. Panfilio $\mathrm{CB}$, Hernández-Cossio $\mathrm{O}$, Hernández $\mathrm{OJ}$ : Orbital myositis and rheumatoid arthritis: case report. Arq Neuropsiquiatr 58(1): 174-7, 2000.

14. Van de Mosselaer G, Deuren HV, Dewolf-Peeters C, et al.: Pseudotumor orbitae and myasthenia gravis. Arch Ophthalmol 98:1621-2, 1980

15. Harris GJ: Idiopatic orbital inflammation: a pathogenic construct and treatment strategy. Ophthal Plastic and Reconstr Surg 22(2): 79-86, 2006.

16. Cervellini P, Volpin L, Curri D, et al.: Sclerosing orbital pseudotumor. Ophthalmologica 193: 39-44, 1986.

17. Rootman $\mathrm{J}, \mathrm{McCarthy} \mathrm{M}$, White $\mathrm{V}$, et al.: Idiopathic sclerosing inflammation of the orbit a distinct clinicopathologic entity. Ophthalmology 101: 570-84, 1994.

18. Cush JJ, Leibowitz IH, Friedman SA: Adult-onset Still's disease and inflammatory orbital pseudotumor. N Y State J Med 85(3): 110-1, 1985.

19. Ganday G, Imbert Y, Coomans D, Leng B: Still's disease in the adult. Sem Hop Paris 57: 1885-9, 1981. 